\title{
Delivering dental care: changing needs and future requirements
}

The dramatic improvements in dental health are a good test bed to evaluate the openness to change in dentistry. Dentistry is fast becoming more feminised. There is a substantial change in the sex ratio of the profession and women have different values and perspectives. Female dentists are more likely to be employed than to be practice owners. However, the existing ownership of the infrastructure for delivery of care is not readily compatible with employment arrangements for many women. Single handed dentists and premises still form the largest component of primary dental care practices. What is more, successful primary dental care depends on long term relationships. Dentists working in primary dental care want secure contracts and long-term commitment by PCTs. But who will commit themselves to the dental needs of a community when the PCT contracts on offer are short-term and the future is filled with uncertainty?

The questions that policy makers must answer are; to what extent do current proposals for care take the abovementioned trends in disease, structure of the profession and contractual arrangements into account? Policies should be structured to enable dentists to make sound decisions on a secure basis to plan their practices and futures. Most importantly, systems of dental care should ensure there are internal incentives and concern for quality, economy, and opportunities to maximise patient service to stimulate good practice. The current proposed dental arrangements do not. They have insufficient incentives: they fail to address the changing trends in diseases, dental needs, feminisation of the workforce and they rely on short term contracts.

The main focus of a strategy should be on devising mechanisms for rewarding dentists so that they focus on reducing treatment need; needs that are based on both non-clinical and clinical measures. Rewards should be linked to outcomes acceptable to patients, care providers and government. Part of the reward mechanism should be the development of a career structure that allows dentists to move from an initial position requiring basic clinical skills, to one with increased advanced clinical and managerial aspects or to a planning level. Such a strategy will be particularly conducive to women's careers and reward female dentists. They will benefit from a flexible career structure and rewards based on reducing treatment needs and limiting intervention to a minimum. Currently they are unjustly criticised for lower 'productivity' yet they are less interventionist a quality that needs encouraging when disease levels are falling.

Analyses of the dental health of Europeans indicate that disease levels are low and decreasing in each new age cohort. Need for dental care will decline further. Most people will require little regular dental care and most interventions will be simple. The content of treatment courses will change. Such trends will continue and accelerate. Furthermore, at low levels of caries and periodontal disease traditional preventive methods cannot be justified. As Dowell said "Recent events have made a more open approach to clinical governance essential if the professions are to recover and retain the confidence of the public." The increasing focus on openness and public scrutiny, clinical governance and the possible requirements for revalidation will profoundly influence provision of public and private dentistry. Selective elimination of inappropriate care following the application of clinical governance would free up resources to provide evidence-based care to more people.

What are required are policies that foster incentives to enhance the quality of the care delivery system. Policies should take into account the changing sex balance and existing ownership of practices. They should centre on reducing need, fulfilling professional careers, rewarding commitment and allow identification of good practice as well as support the attainment of high standards. All hallmarks of a quality health care system. 